\title{
ASOCIACIÓN ENTRE DISMENORREA PRIMARIA Y AUSENTISMO ACADÉMICO EN ESTUDIANTES DE MEDICINA DE PRIMER Y SEGUNDO AÑO DE LA UNIVERSIDAD RICARDO PALMA EN JUNIO DEL 2016
}

\author{
ASSOCIATION BETWEEN PRIMARY DISMENORRHEA AND ACADEMIC ABSENTEEISM IN STUDENTS OF \\ MEDICINE FROM FIRST AND SECOND YEAR OF RICARDO PALMA UNIVERSITY IN JUNE 2016 \\ Scarlet Oderay Santa Cruz-Rojas', Lucy E. Correa-López²,3
}

\begin{abstract}
RESUMEN
La dismenorrea primaria es muy frecuente; a nivel mundial puede presentarse en un $90 \%$ en adolescentes y en más del $50 \%$ de las mujeres que tienen menstruación; entre el $10 \%$ y $20 \%$ de ellas dicen que el dolor puede llegar a ser insoportable. Objetivos: Determinar la asociación entre dismenorrea primaria y ausentismo académico en estudiantes de medicina de primer y segundo año de la Universidad Ricardo Palma en Junio del 2016. Métodos: Estudio observacional, cuantitativo, analítico - relacional y transversal. Se encuestaron a 249 estudiantes de medicina de primer y segundo año de la Universidad Ricardo Palma. Resultados: El 57,03\% de las estudiantes encuestadas indica que por causa del dolor menstrual tuvieron ausentismo académico, y un 30,52\% a pesar de tener dolor no presenta ausentismo académico $\left(x^{2}=8,033 ; p=0.005 ; O R=2,958 I C=1.364-6,418\right)$. Conclusión: La dismenorrea en la mayor parte de las estudiantes ha sido causante de afectación en las actividades diarias, demostrando que existe asociación significativa entre dismenorrea primaria y ausentimo académico.
\end{abstract}

Palabras clave: Dismenorrea primaria; Ausentismo académico; Estudiantes de medicina. (fuente: DeCS BIREME)

\begin{abstract}
Primary dysmenorrhea is very common; in the world, it can be present in $90 \%$ of the adolescents and in more than $50 \%$ of the women who have menstruation, and $10-20 \%$ of them say that the pain can become unbearable. Objective: Determine the association between primary dysmenorrhea and academic absenteeism in first and second year medical students of the Ricardo Palma University in June, 2016. Methods: Observational, quantitative, analytical - relational and transversal study. 249 students from the first and second year of the medical faculty from the Ricardo Palma University were surveyed. Results: $57,03 \%$ of the students surveyed presented academic absenteeism when presenting menstrual pain, however; $30,52 \%$ despite having pain didn' $t$ present academic absenteeism ( $\left.x^{2}=8,033 ; p=0.005 ; O R=2,958 \mathrm{IC}=1.364-6,418\right)$. Conclusions: Dysmenorrhoea in most part of the students has been cause of affectation in daily activities, demonstrating that there is a significant association between primary dysmenorrhoea and academic absenteeism.
\end{abstract}

Key words: Primary dysmenorrhea; Academic absenteeism; Medical students. (source: MeSH NLM)

\footnotetext{
' Facultad de Medicina Humana, Universidad Ricardo Palma, Lima, Perú.

2 Instituto de Investigación en Ciencias Biomédicas, Universidad Ricardo Palma, Lima, Perú.

${ }^{3}$ Economista, Master of Business Administration.
}

Correspondencia: Scarlet Oderay Santa Cruz Rojas. Dirección: Jr. Cuzco 456 dpto 602. Magdalena, Lima - Perú. Teléfono: 51-962-370-310. Correo: telracs2069@hotmail.com

Citar como: Scarlet Oderay Santa Cruz-Rojas, Lucy E. Correa-López. Asociación entre dismenorrea primaria y ausentismo académico en estudiantes de medicina de primer y segundo año de la Universidad Ricardo Palma en junio del 2016. [Artículo Original]. Rev. Fac. Med. Hum. 2017;17(1):64-71. DOI 10.25176/RFMH.v17.n1.750 


\section{INTRODUCCIÓN}

La dismenorrea primaria es muy frecuente, principalmente en mujeres jóvenes que se encuentran en la adolescencia. En el mundo, puede presentarse hasta en un $90 \%$ de las adolescentes y en más del $50 \%$ de las mujeres que tienen menstruación. Además entre el $10-20 \%$ de ellas dicen que el dolor es intenso y angustiante $\mathrm{e}^{1,2,3,4}$. La dismenorrea tiene un efecto negativo en el desempeño cotidiano de las mujeres que la padecen. La presentación clínica es de grado de intensidad variable y puede llegar a ser incapacitante, ocasionando problemas de ausentismo, disminución del rendimiento académico, alteraciones en el estado deánimoy afectación de las relaciones interpersonales. Se ha reportado que existe entre un 10-15\% de ausentismo escolar o laboral por esta causa, y un 5\% de incapacidad para la adecuada realización de las actividades diarias ${ }^{5,6,7,8}$.

Estudios realizados en Estados Unidos en la Universidad de Florida, señalan que al año se pierden alrededor de 600 millones de horas laborales y 2.000 millones de dólares a causa de la dismenorrea primaria. Además, las mujeres que trabajan mientras sufren de dolor menstrual tienen menor productividad y menor calidad en el desempeño de sus actividades ${ }^{6,7,9}$.

La prevalencia de dismenorrea primaria en adolescentes (10-19años deacuerdo con la clasificación de la OMS) varía entre el 51,3\% y el $85 \%$, de acuerdo a las características demográficas de las poblaciones incluidas en los diferentes estudios ${ }^{10,11,12,13,14}$.

En el Perú, por logeneral los investigadores asumen que la dismenorrea es un síntoma "normal" que acompaña la menstruación, y esto debido a la frecuencia de su aparición y además consideran que esta frecuencia será semejante a las estadísticas recogidas en otras poblaciones ${ }^{15}$.

Según un estudio realizado en el 2006 en LimaPerú, la prevalencia de dismenorrea en población universitaria fue de $88.7 \%{ }^{16}$, por lo que siendo estas tasas de frecuencia alarmantes y pese a que no es una patología mortal, es importante conocer acerca de dismenorrea en nuestro medio para evitar posibles repercusiones asociadas a este problema en el futuro.

\section{MÉTODOS}

Se realizó un estudio de tipo observacional, cuantitativo, analítico - relacional y transversal.

La población de estudio estuvo conformada por 249 estudiantes de medicina de primer y segundo año que estuvieron matriculadas en la Universidad Ricardo Palma en el I semestre del año 2016, además en la presente investigación no se consideró una muestra probabilística pues se recolectaron los datos de todas las estudiantes de la población. La unidad de análisis fue estudiante mujer que cursaba el primer o segundo año de medicina en la Universidad Ricardo Palma en junio del 2016, quién a través de una encuesta que llenó, brindó los datos necesarios para la consecución de los objetivos del presente estudio. Se empleó el cuestionario de dolor menstrual propuesto por Larroy ${ }^{17}$, quien determinó el valor discriminativo de los elementos del cuestionario mediante una prueba a de Cronbach encontrándose que todos los elementos (ítems) relevantes eran capaces de diferenciar entre personas con o sin trastorno (dismenorrea) a un nivel de confianza del $99 \%$. Los datos fueron procesados utilizando el programa estadístico spss v22. Se utilizaron medidas de asociación estadística como chi cuadrado y Odds ratio.

\section{RESULTADOS}

De las 249 estudiantes encuestadas se halló que 60 pertenecían al I ciclo; 82 al II ciclo; 49 al ciclo III y 58 al IV ciclo, la mayor cantidad de estudiantes encuestadas pertenecían al II ciclo como se muestra en la tabla 1.

Tabla 1. Ciclo Académico.

\begin{tabular}{lcc}
\hline & Frecuencia & Porcentaje \\
\hline I ciclo & 60 & 24,1 \\
\hline II ciclo & 82 & 32,9 \\
\hline III ciclo & 49 & 19,7 \\
\hline IV ciclo & 58 & 23,3 \\
\hline Total & 249 & 100,0 \\
\hline
\end{tabular}

Fuente: INICIB-FAMURP

Entre las características encontradas en las estudiantes podemos destacar que en cuanto al estado civil la mayoría son solteras y muy pocas son convivientes. Algo similar ocurre en cuanto a paridad pues un $97,59 \%$ no han tenido partos y solo un $2,41 \%$ han tenido hijos. En cuanto al inicio de relaciones sexuales un $40,96 \%$ afirman ya haber iniciado su vida sexual y un $59,04 \%$ no lo refiere. En cuanto al uso de anticonceptivos un $38,6 \%$ afirman ya usarlos y un $61,4 \%$ no los usan. 
Con respecto a la menarquia un 46,59\% refiere haberla presentado a los 12 años. En el 30,52\% de las estudiantes la duración de la menstruación es 4 días y en el $36,55 \%$ es de 5 días. En cuanto a la duración del ciclo menstrual la mayoría presenta un promedio de 28 días por ciclo. Finalmente, referencia a la cantidad de sangrado en cada menstruación, el 16,87\% presenta una cantidad leve, $77,91 \%$ moderada y solo un $5,22 \%$ severa (tabla 2).

Tabla 2. Características demográficas y clínicas de las estudiantes.

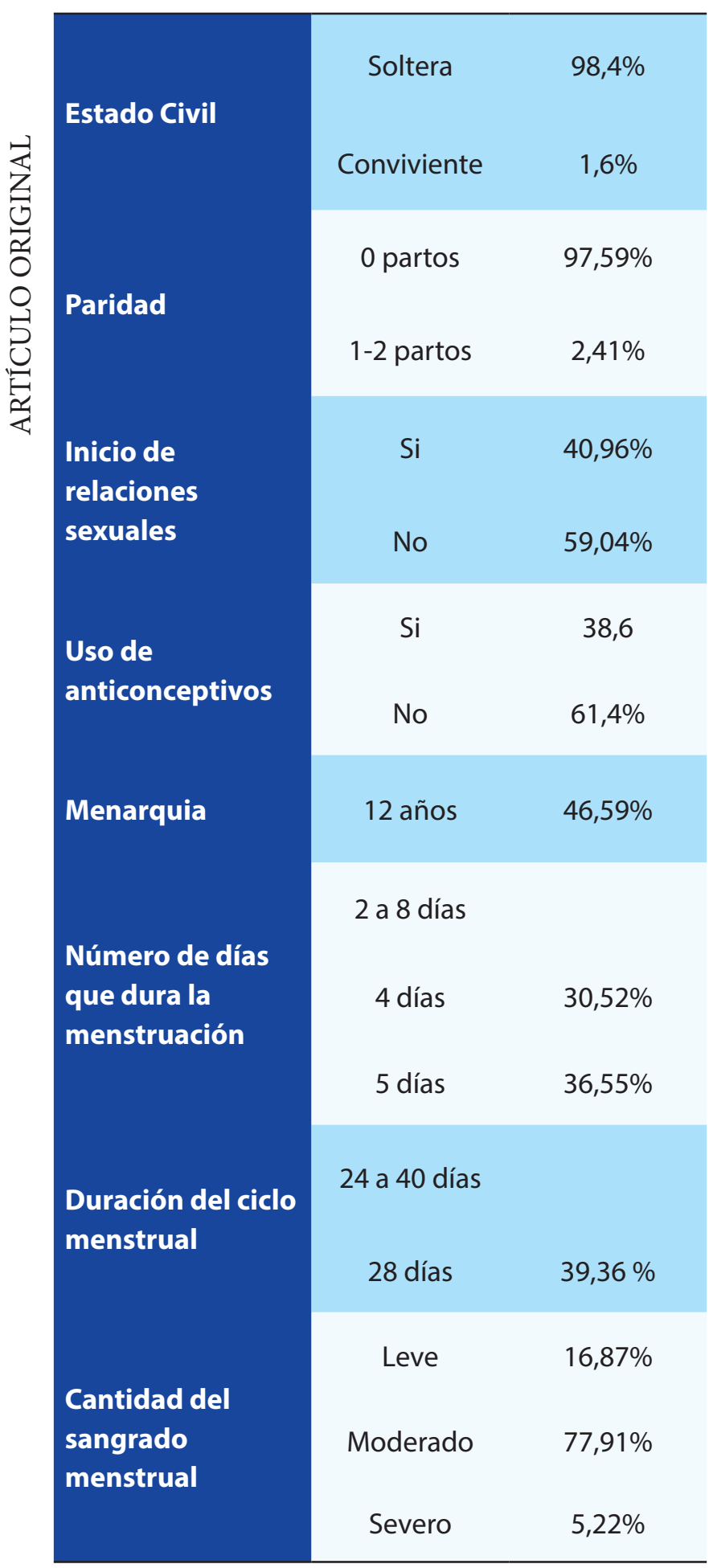

Fuente: INICIB-FAMURP
Entre los casos de dismenorrea primaria en las estudiantes un $12,4 \%$ no refiere tener dolor mientras que un $87,6 \%$ sí dice presentarlo, dichos resultados se aprecian en la tabla 3.

Tabla 3. Dismenorrea primaria

\begin{tabular}{lcc}
\hline & Frecuencia & Porcentaje \\
\hline Hay dolor & 218 & 87,6 \\
\hline No hay Dolor & 31 & 12,4 \\
\hline Total & 249 & 100,0 \\
\hline
\end{tabular}

Fuente: INICIB-FAMURP

Con respecto al grado de severidad de dismenorrea primaria el $12,45 \%$ de las encuestadas afirmaron que no tenían dolor, $28,92 \%$ tenían dismenorrea leve, $46,59 \%$ dismenorrea moderada y $12,05 \%$ dismenorrea severa (gráfico 1).

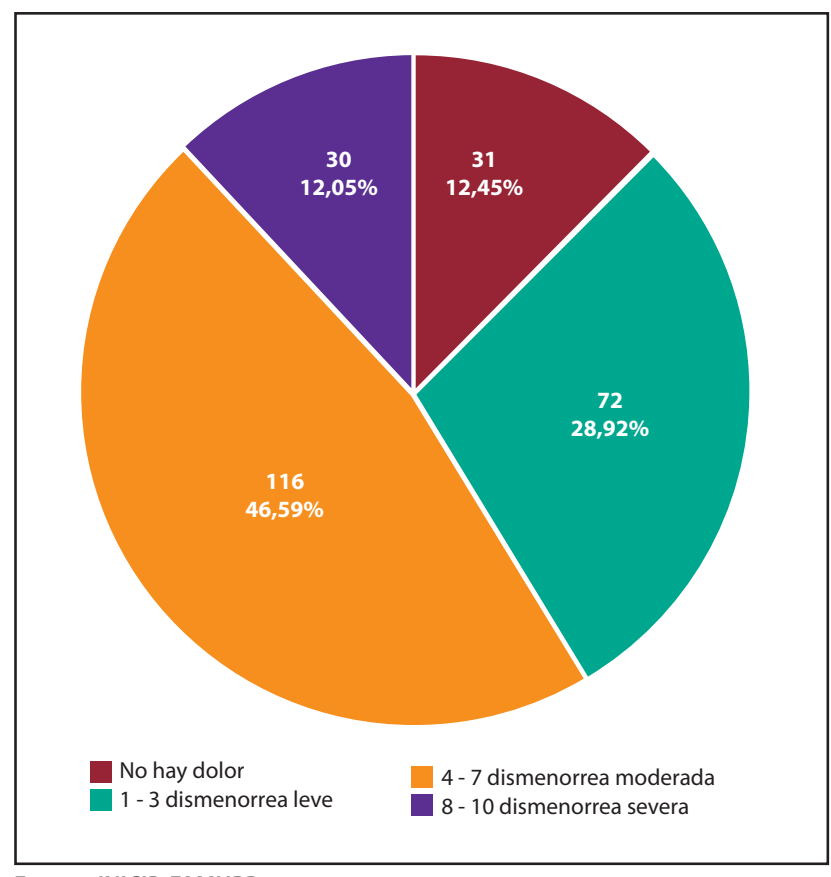

Fuente: INICIB-FAMURP

Gráfico 1. Grado de dismenorrea primaria.

Se observa en la tabla 4, que el 57,03\% de las estudiantes encuestadas sí presentan ausentismo académico al presentar el dolor menstrual, sin embargo; un $30,52 \%$ a pesar de tener dolor no presenta ausentismo académico. Un 4,82\% presenta ausentismo, aunque no tiene dolor y 7,63\% no presenta ni dolor ni ausentismo.

Además, el valor de $\mathrm{x}^{2}=8,033$ y $\mathrm{p}=0.005(<0.05)$ para la asociación entre dismenorrea y ausentismo académico, encontrándose significancia estadística, además siendo el Odds Ratio mayor a la unidad $(\mathrm{OR}=2,958)$ decimos que las estudiantes que presentan dismenorrea primaria tienen más probabilidad de presentar ausentismo académico. 
Tabla 4. Ausentismo académico vs dismenorrea primaria

\begin{tabular}{cccc} 
& \multicolumn{3}{c}{ Dismenorrea Primaria } \\
& & Sí & No \\
\hline $\begin{array}{c}\text { Ausenteismo } \\
\text { Académico }\end{array}$ & Si & 142 & 12 \\
& No & $7,03 \%$ & $4,82 \%$ \\
& & $30,52 \%$ & $7,63 \%$ \\
\hline
\end{tabular}

\begin{tabular}{cccccc}
\hline & & & \multicolumn{2}{c}{ IC al 95\% } \\
& Chi & $p$ & OR & $\begin{array}{c}\text { Límite } \\
\text { inferior }\end{array}$ & $\begin{array}{c}\text { Límite } \\
\text { superior }\end{array}$ \\
\hline & cuadrado & & & & \\
\hline & 8,033 & 0,005 & 2,958 & 1,364 & 6,418 \\
\hline
\end{tabular}

Fuente: INICIB-FAMURP

En la tabla 5 se muestra que dentro de los casos de dismenorrea leve un 10,04\% presenta ausentismo académico y un $18,88 \%$ aunque refiere dolor no presenta ausentismo académico; además entre los casos que no presentan dismenorrea leve un 51,81\% sí presenta ausentismo académico y un $19,28 \%$ no lo presenta. En cuanto a la dismenorrea moderada un
$37,35 \%$ presenta ausentismo y un $8,84 \%$ a pesar de tener dismenorrea no refiere ausentismo y dentro de los casos que no presentan dismenorrea moderada un $24,50 \%$ sí presenta ausentismo académico y un $29,32 \%$ no lo presenta. Finalmente, en el grupo de dismenorrea severa un $10,44 \%$ presenta ausentismo académico y tan solo un $1,61 \%$ no presenta ausentismo, aunque sí tiene dismenorrea severa además entre los casos que no presentan dismenorrea severa un 51,41\% sí presenta ausentismo académico y un $36,55 \%$ no refiere presentarlo.

Además, el valor del $x^{2}=31,583$ y $p=0.001(<0.05)$ encontrándose significancia estadística para el factor dismenorrea leve asociado a ausentismo académico, pero al ser el Odds Ratio menor a la unidad $(\mathrm{OR}=0,198)$ decimos que la dismenorrea leve sería un factor protector contra el ausentismo académico. Por otro lado, se halló un $\mathrm{x}^{2}=32,769$ y $\mathrm{p}=0.001(<0.05)$ encontrándose significancia estadística para el factor dismenorrea moderada asociado a ausentismo académico y al ser el Odds Ratio mayor a la unidad $(\mathrm{OR}=5,059)$ decimos que la dismenorrea moderada es un factor de riesgo para ausentismo académico. Algo similar se encontró en la asociación entre dismenorrea severa con ausentismo académico encontrándose un $x^{2}=8,904$ y $p=0.003(<0.05)$ con significancia estadística; y al ser el Odds Ratio mayor a la unidad $(\mathrm{OR}=4,621)$ decimos que la dismenorrea severa es un factor de riesgo para el ausentismo académico.

Tabla 5. Ausentismo académico vs grado de dismenorrea primaria.

\begin{tabular}{|c|c|c|c|}
\hline & & \multicolumn{2}{|c|}{ DISMENORREA LEVE } \\
\hline & & Sí & No \\
\hline \multirow{4}{*}{ AUSENTISMO ACADÉMICO } & \multirow{2}{*}{$\mathrm{Si}$} & 25 & 129 \\
\hline & & $10,04 \%$ & $51,81 \%$ \\
\hline & \multirow{2}{*}{ No } & 47 & 48 \\
\hline & & $18,88 \%$ & $19,28 \%$ \\
\hline & & \multicolumn{2}{|c|}{ DISMENORREA MODERADA } \\
\hline & & Sí & No \\
\hline \multirow{4}{*}{ AUSENTISMO ACADÉMICO } & \multirow{2}{*}{ Si } & 93 & 61 \\
\hline & & $37,35 \%$ & $24,50 \%$ \\
\hline & \multirow{2}{*}{ No } & 22 & 73 \\
\hline & & $8,84 \%$ & $29,32 \%$ \\
\hline & & \multicolumn{2}{|c|}{ DISMENORREA SEVERA } \\
\hline & & Sí & No \\
\hline \multirow{4}{*}{ AUSENTISMO ACADÉMICO } & \multirow{2}{*}{$\mathrm{Si}$} & 26 & 128 \\
\hline & & $10,44 \%$ & $51,41 \%$ \\
\hline & \multirow{2}{*}{ No } & 4 & 91 \\
\hline & & $1,61 \%$ & $36,55 \%$ \\
\hline
\end{tabular}

Fuente: INICIB-FAMURP 


\begin{tabular}{llllll}
\multicolumn{1}{c}{ Factor } & Chi cuadrado & P & OR & \multicolumn{2}{c}{ IC al 95\% } \\
\hline Lismenorrea leve & 31,583 & 0,001 & 0,198 & 0,110 & 0,356 \\
\hline Dismenorrea moderada & 32,769 & 0,001 & 5,059 & 2,844 & 8,997 \\
\hline Dismenorrea severa & 8,904 & 0,003 & 4,621 & 1,559 & 13,695 \\
\hline
\end{tabular}

Fuente: INICIB-FAMURP

Entre las estudiantes que presentaron dismenorrea primaria, 57,83\% vieron afectadas sus actividades diarias debido al dolor, pero un $29,72 \%$ a pesar de presentar dolor no tuvieron alteración de sus actividades diarias. Por otro lado, un 4,02\% refiere que, aunque no presenta dismenorrea si ve afectadas sus actividades diarias por otros motivos y otro $8,43 \%$ que no presenta dismenorrea tampoco refiere alteración de sus actividades diarias.

Además, se obtuvo $x^{2}=13,138$ y $p=0.001(<0.05)$ para la asociación entre actividades diarias y dismenorrea primaria, encontrándose significancia estadística, y el OR al ser mayor a la unidad $(\mathrm{OR}=4,086)$ nos dice que las estudiantes tienen más riesgo de presentar afectación de las actividades diarias debido a la presencia de dismenorrea primaria (tabla 6).

Tabla 6. Dismenorrea primaria vs actividades diarias.

\begin{tabular}{cccc}
\hline & \multicolumn{2}{c}{ Dismenorrea Primaria } \\
& \multicolumn{1}{c}{ Sí hay dolor } & $\begin{array}{c}\text { No hay } \\
\text { dolor }\end{array}$ \\
\hline $\begin{array}{c}\text { Actividades } \\
\text { Diarias }\end{array}$ & $\begin{array}{c}\text { Sí hay } \\
\text { Afectación }\end{array}$ & 144 & 10 \\
& $\begin{array}{c}\text { No hay } \\
\text { Afectación }\end{array}$ & $74,83 \%$ & $4,02 \%$ \\
\hline
\end{tabular}

\begin{tabular}{ccccc}
\hline Chi & IC al 95\% & OR & $\begin{array}{c}\text { Límite } \\
\text { inferior }\end{array}$ & $\begin{array}{c}\text { Límite } \\
\text { superior }\end{array}$ \\
cuadrado & & & 1,830 & 9,127 \\
\hline 13,138 & 0,001 & 4,086 & . & \\
\hline
\end{tabular}

Fuente: INICIB-FAMURP

De las estudiantes que presentan dismenorrea un $55,02 \%$ llega a automedicarse y un $32,53 \%$ no toma ningún medicamento. Dentro del grupo de estudiantes que no presentan dismenorrea, un 3,61\% aunque no refiere dismenorrea sí se ha automedicado por otros motivos y un $8,84 \%$ al no presentar dolor no presenta tampoco automedicación.

Además, vemos que se obtuvo el valor de $x^{2}=$ 12,793 y $\mathrm{p}=0.001 \quad(<0.05)$ para la asociación de automedicación con dismenorrea primaria encontrándose significancia estadística y siendo el OR mayor a la unidad $(O R=4,134)$ podemos decir que las estudiantes que presentan dismenorrea tienen más riesgo de automedicarse (tabla 7).

Tabla 7. Dismenorrea primaria vs automedicación.

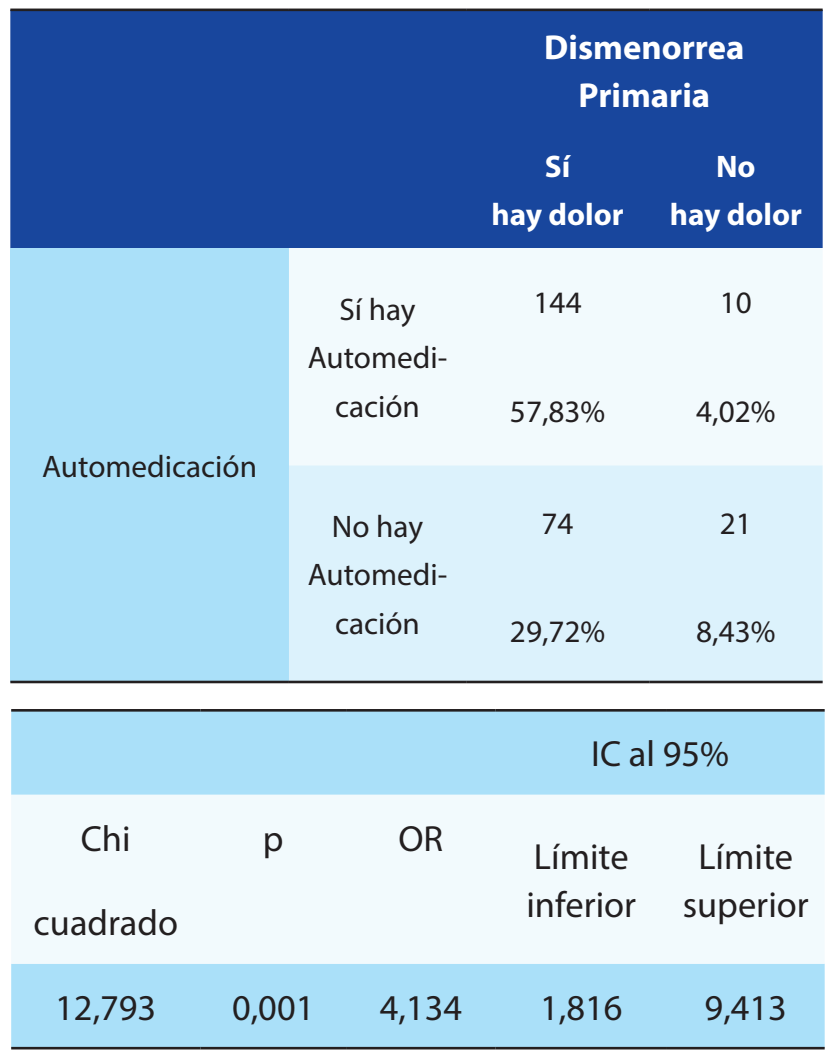

Fuente: INICIB-FAMURP

De las 249 universitarias, un $71,49 \%$ de las estudiantes con dismenorrea son menores de 21 años y solo un $16,06 \%$ presentan dismenorrea primaria de las estudiantes mayores de 21 años. Un 6,43\% de las estudiantes menores de 21 años refiere no presentar dismenorrea y un $6,02 \%$ de las mayores de 21 años dice también no presentarla (gráfico 2). 


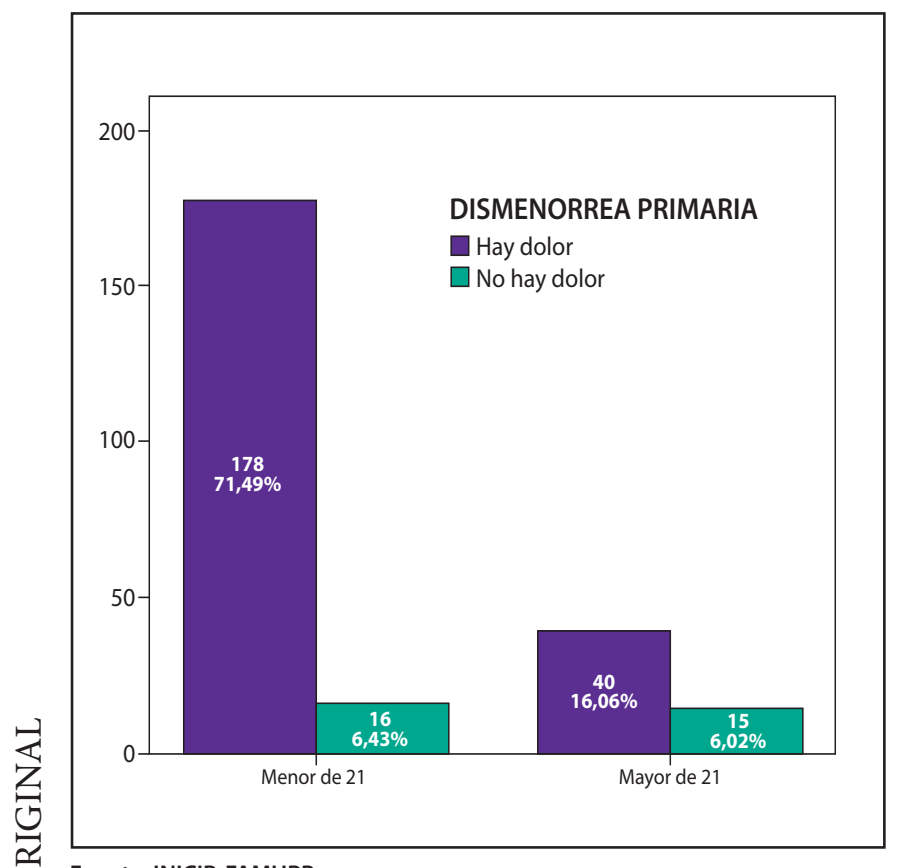

Fuente: INICIB-FAMURP

Gráfico 2. Asociación entre dismenorrea primaria y edad.

\section{DISCUSIÓN}

La dismenorrea es uno de los trastornos ginecológicos más comunes que se ve con mayor frecuencia en mujeres jóvenes y al presentar una alta prevalencia podríamos decir que estamos frente a una patología de salud pública. Sin embargo, no se le da la debida importancia y por ende las afectadas suelen recurrir solo a la automedicación para tratar el dolor.

Así mismo, la dismenorrea puede ser causa de afectación de actividades diarias en las mujeres y ya que un gran número de ellas sufre de esta patología y debido a que la mayoría se encuentra en actividad académica o laboral constante, son muchas las horas de trabajo y estudio que se pierden a consecuencia de la dismenorrea, y no solo ello, sino que también pueden verse afectadas la concentración y el rendimiento de las mujeres lo cual les condiciona a tener desventajas frente al ámbito laboral y estudiantil y es por ello que se deberían crear programas para información de la población con respecto a esta patología y así poder prevenir repercusiones negativas en la vida de las mujeres que la padecen.

Según la edad en la que con mayor frecuencia se presenta la dismenorrea primaria, Dalton sostiene que la edad oscila entre los 15 y 25 años de edad, luego de lo cual va desapareciendo gradualmente. Es por ello que en nuestro estudio utilizamos a una población con una edad promedio de 19,65 años ya que en este grupo etáreo se puede estudiar de manera más específica las posibles repercusiones que el dolor menstrual pueda ocasionar en la vida de las jóvenes que lo padecen, además podemos relacionar lo que dice Dalton con uno de los resultados obtenidos en este estudio pues pudimos ver que en las estudiantes menores de 21 años existen un mayor número de casos de dismenorrea primaria a diferencia de aquellas estudiantes mayores de 21 años.

En cuanto a la prevalencia de la dismenorrea primaria, en múltiples estudios estadísticos se señala que más del $50 \%$ de las adolescentes la presentan, pudiendo alcanzar a un $90 \%$ de la población femenina comprendida en el grupo etáreo ya mencionado. En este estudio, la dismenorrea primaria tuvo una prevalencia del $87,6 \%$ en las estudiantes de primer y segundo año de medicina, similar a los porcentajes anteriormente mencionados. Banikam en su estudio realizado en adolescentes hispanas en el 2000 encontró una prevalencia de dismenorrea de $85 \%$ y porcentaje similar de $80 \%$ se obtuvo en el trabajo que Hillen realizó en adolescentes australianas.

Con respecto al grado de dismenorrea primaria, se ve que cuanto más severa, más influirá en los aspectos de la vida cotidiana de las afectadas, diversos autores mencionan que el grado de dismenorrea severa puede variar de una población a otra, pudiendo encontrarse porcentajes de hasta 45\%, como en el trabajo realizado por Hoyos en el 2010 en donde se encontró una prevalencia de dismenorrea severa del 33,78\%; sin embargo, en este estudio que presentamos se encontró una prevalencia de dismenorrea severa de $12,05 \%$ en cambio la dismenorrea moderada llego a alcanzar un porcentaje de $46,59 \%$ y la dismenorrea leve fue de $28,92 \%$. También se pudo comprobar que cuanto mayor sea la severidad de dismenorrea que presentan las estudiantes, mayor será la relación que tenga con el ausentismo académico.

Al ser la dismenorrea primaria una patología muy frecuente, llega a afectar en gran medida la vida de las que la presentan, por ello en este estudio se analizó el efecto que tiene sobre la asistencia a clases universitarias de las estudiantes, hallándose que en un $57,03 \%$ de la población de estudio debido al dolor menstrual presentó ausentismo académico. Jiménez $y$ cols nos dicen que en el trabajo que realizaron un $61,6 \%$ de las universitarias presentaron problemas a causa de la dismenorrea y que un $35.7 \%$ presentó ausentismo académico debido al dolor.

En la presente investigación también se analizó la asociación entre la dismenorrea y la alteración de las actividades diarias de las estudiantes, obteniendo como resultado que un $57,83 \%$ de las que presentan dismenorrea ven afectadas sus actividades diarias 
de manera negativa y tan solo un $29,72 \%$ no refiere ningún tipo de alteración en su vida diaria debido al dolor menstrual. Bazalar en su investigación del 2014 obtiene los siguientes datos con respecto a la dismenorrea y alteración de las labores diarias de su población de estudio: $79.5 \%$ de las internas de obstetricia y $84.6 \%$ de las internas de nutrición presentaron afectación de sus actividades debido al dolor menstrual, porcentajes superiores al hallado en nuestro estudio.

Actualmente existen nuevos descubrimientos con respecto a la etiología de los dolores menstruales y su tratamiento. Pero como vimos en nuestros resultados, la mayoría de las estudiantes encuestadas (55,02\%) llega a automedicarse para disminuir el dolor sin acudir antes a consultar a un especialista.

Existen una gran cantidad de cosas por estudiar con respecto a la dismenorrea primaria, y se espera que el estudio presentado pueda contribuir para el desarrollo de futuros estudios con respecto a este tema y más investigadores se interesen por el tópico aquí tratado y de esta manera se puedan establecer mejores políticas de salud con respecto a la dismenorrea y se le dé la debida importancia que requiere.

\section{CONCLUSIÓN}

La dismenorrea primaria es una patología más común de lo que se piensa, ya que en nuestro estudio realizado tuvo una frecuencia de $87,6 \%$.

En la Universidad Ricardo Palma de las estudiantes de medicina d primer y segundo año, el 57,03\% de las estudiantes presenta ausentismo académico al presentar dismenorrea encontrándose una asociación significativa, $\left(x^{2}=8,033 ; p=0,005\right)$ y al ser el $\mathrm{OR}=2,958$ concluimos que la dismenorrea primaria es factor de riesgo para ausentismo académico en estudiantes de medicina de primer y segundo año de la Universidad Ricardo Palma.

Cuanto mayor sea el grado de dismenorrea más serán los casos en los que se presente ausencia a clases, ya que de las alumnas que presentan dismenorrea leve, solo un $10,04 \%$ han faltado hallándose un $x^{2}=31,583$ y $p=$ 0.001 (< 0.05$)$ encontrándose significancia estadística pero al ser el $\mathrm{OR}=0,198$, la dismenorrea leve sería un factor protector contra el ausentismo académico, a diferencia de las alumnas que presentan dismenorrea moderada las cuales en un $37,35 \%$ se han ausentado al presentar dolor menstrual encontrándose un $x^{2}=$ 32,769 y $p=0.001(<0.05)$ encontrándose significancia estadística y al ser el $\mathrm{OR}=5,059$ decimos que la dismenorrea moderada es un factor de riesgo para ausentismo académico y lo mismo ocurren en el caso de la dismenorrea severa con un $x^{2}=8,904$ y $p=0.003$ $(<0.05)$ con significancia estadística; $y$ un $\mathrm{OR}=4,621$.

La dismenorrea en la mayor parte de las estudiantes ha sido causante de afectación en las actividades diarias, presentándose en un $57,83 \%$ de las encuestadas encontrándose así una asociación significativa, $\left(x^{2}=13,138 ; p=0,001\right)$ y al ser el $O R=4,086$ decimos que la dismenorrea primaria es factor de riesgo para afectación de las actividades diarias.

Un 55,02\% de las estudiantes con dismenorrea primaria se automedica para aliviar su dolor y un $32,53 \%$ a pesar de tener dolor no toma ningún tipo de fármaco. Al ser el $x^{2}=12,793 ; p=0,001$, la asociación entre automedicación y dismenorrea es significativa y al hallar un $\mathrm{OR}=4,134$, concluimos que la dismenorrea primaria es factor predisponente para automedicación en las estudiantes de medicina de primer y segundo año de la universidad Ricardo Palma.

Las estudiantes menores de 21 años tienen mayor riesgo de presentar ausentismo académico debido a dismenorrea primaria a diferencia de aquellas mayores de 21 años, pues en este estudio se encontró un 71,49\% de estudiantes menores de 21 años que presentaban dismenorrea $\left(x^{2}=14.230 ; p=0.001(<0.05) ; O R=4,172\right)$.

Financiamiento: Autofinanciado.

Conflicto de interés: Los autores declaran no tener conflictos de interés en la publicación de este artículo.

Recibido: 06 de marzo del 2017

Aprobado: 27 de marzo del 2017 


\section{REFERENCIAS BIBLIOGRÁFICAS}

1. Davis $A R$, Westhoff $C L$. Primary dysmenorrhea in adolescent girls and treatment with oral contraceptives. J Pediatr Adolesc Gynecol 2001; 14:3-8.

2. Durain D. Primary dysmenorrhea: assessment and management update. J Midwifery Womens Health 2004; 49:520-8.

3. Ortiz MI, Rangel-Flores E, Carrillo-Alarcón LC, Veras-Godoy HA. Prevalence and impact of primary dysmenorrhea among Mexican high school students. Int J Gynaecol Obstet 2009; 107:240-3.

4. Fedorowicz Z, Nasser M, Jagannath VA, Beaman JH, Ejaz K, van Zuuren EJ. Beta-2-adrenoceptor agonists for dysmenorrhoea. Cochrane Database Syst Rev 2012;5:CD008585

5. Banikarim C, Chacko M, Kelder S. Prevalence and impact of dysmenorrheal on hyspanic female adolescents. Arch Pediatr Adolesc Med 2000; 154: 1226-9.

6. Polat A, Celik H, Gurates B, Kaya D, Nalbant M, Kavak E, et al. Prevalence

of primary dysmenorrhea in young adult female university students. Arch Gynecol Obstet 2009; 279: 527-32.

7. Pullon S, Reinken J, Sparrow M. Prevalence of dysmenorrheal in Wellington women. NZ Med J 1988;101: 52-4.

8. Yáñez N., Bautista S., Ruiz J., Ruiz A. Prevalencia y factores asociados a dismenorrea en estudiantes de ciencias de la salud. Rev. Cienc. Salud 2010; 8 (3): 37-48.

9. Berkley, K. J. Dismenorrea primaria: una necesidad urgente 2013.

10. Yáñez N., Bautista S., Ruiz J., Ruiz A. Prevalencia y factores asociados a dismenorrea en estudiantes de ciencias de la salud. Rev. Cienc. Salud 2010; 8 (3): 37-48.)
11. Hillen TIJ, et al. Primary dysmenorrheal in young western Australian women: prevalence, impact, and knowledge of treatment. J Adolesc Health 1999; 25: 40-5.

12. Ng TP, Tan NCK, Wansaicheong GK. A prevalence study of dysmenorrheal in female residents aged 15-54 years in Clementi Town Singapure. Ann Acad Med Singapure 1992; 21: 323-7.

13. Andersch B, Milsom I. An epidemiologic study of young women with dysmenorrheal. AM J Obstet Gynecol 1982; 144: 655-60.

14. Gurel H, Gurel SA. Dyspareunia, back pain, and chronic pelvic pain: the importance of this pain complex in gynecological practice and its relation with grandmultiparity and pelvic relaxation. Gynecol Obstet Invest 1999; 48: 119-22.

15. Hoyos Rimarachín, C. A. Severidad de la dismenorrea y ausentismo académico en estudiantes de la Universidad Nacional de Trujillo en el año 2009.

16. Roa Y. Dismenorrea y actitudes hacia la propia sexualidad en jóvenes universitarias de Lima-Perú. Rev. Perú. Obstet. Enferm. 2006 jul.-dic.; 2(2):92-98

17. Larroy C, Crespo M y Meseguer C. Funtional dysmenorrhea in the Autonomous Community of Madrid: study of prevalence. Rev. Soc. Esp. Dolor. 2001; 8: 11-22

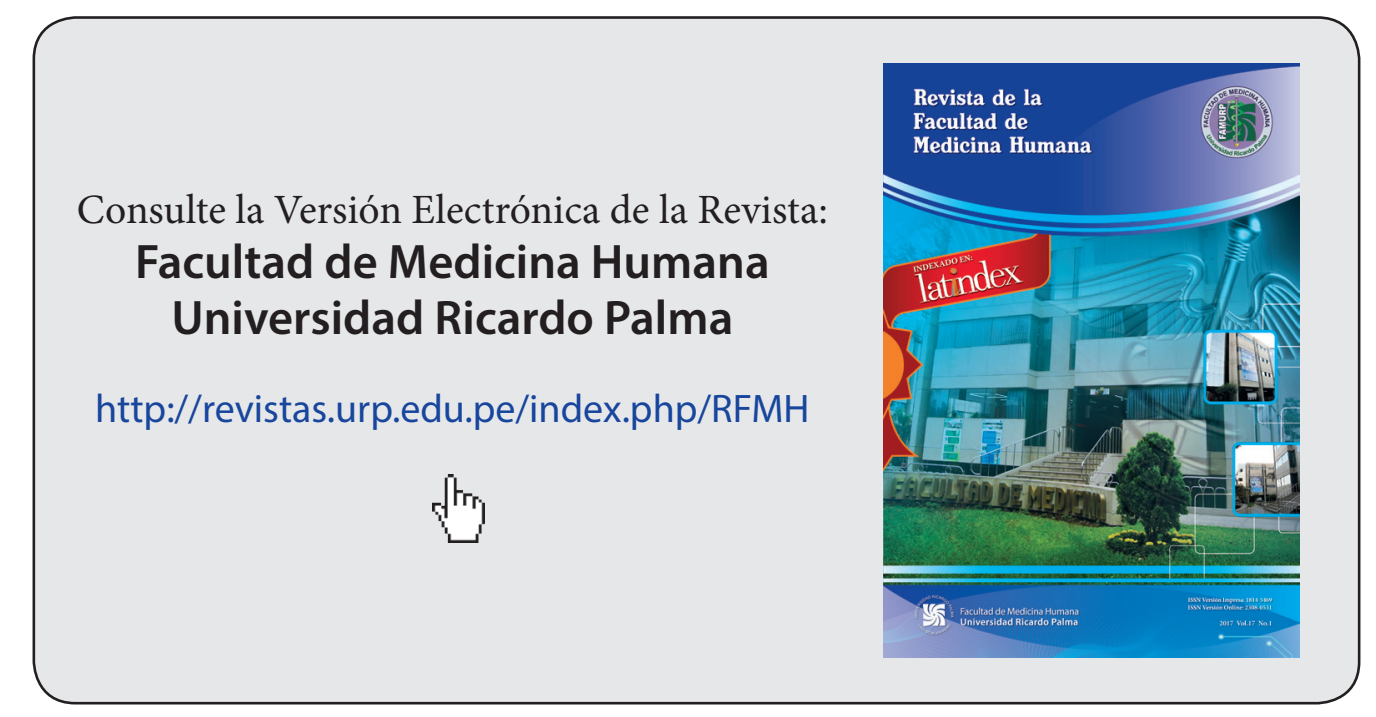

